\title{
Combined Array for Research in Millimeter-wave Astronomy
}

\section{Douglas Bock* and the CARMA Team}

Combined Array for Research in Millimeter-wave Astronomy

E-mail: dbock@mmarray • org

The Combined Array for Research in Millimeter-wave Astronomy (CARMA) has been formed from the millimeter-wave antennas of the Owens Valley Radio Observatory (OVRO) and the Berkeley-Illinois-Maryland Association (BIMA) Array. CARMA consists of six 10.4-m and nine 6.1-m diameter antennas on a site at elevation $2200 \mathrm{~m}$ in the Inyo Mountains of eastern California. The array is operated by an association that includes the California Institute of Technology and the Universities of California (Berkeley), Illinois (Urbana-Champaign), and Maryland. The University of Chicago's Sunyaev-Zel'dovich Array (SZA) is expected to join CARMA with eight 3.5-m antennas in 2008. CARMA has been conducting community 3-mm observations since the beginning of 2007 in two of the five antenna configurations. Commissioning observations at 1.3 $\mathrm{mm}$ and using configurations containing very short $(8 \mathrm{~m})$ and long $(1.7 \mathrm{~km})$ baselines are underway. I present some of the first scientific results from CARMA and describe our program of continuing enhancements.

From Planets to Dark Energy: the Modern Radio Universe October 1-5 2007

The University of Manchester, UK

\footnotetext{
${ }^{*}$ Speaker.
} 


\section{Introduction}

The Combined Array for Research in Millimeter-wave Astronomy (CARMA) has been constructed from nine of the 6.1-m antennas of the Berkeley-Illinois-Maryland Association (BIMA) array [1] and the six 10.4-m antennas of Caltech's Owens Valley Radio Observatory (OVRO) millimeter array [2]. The new array is operated by an association that includes Caltech and the Universities of California (Berkeley), Illinois (Urbana-Champaign), and Maryland. The University of Chicago has recently constructed the Sunyaev-Zel'dovich Array (SZA) of eight 3.5-m antennas at OVRO. In 2008 these antennas will be moved to join CARMA. As well as providing the community with a premier millimeter-wave imaging instrument, CARMA will enable the education of the next generation of millimeter astronomers through hands-on observational and technical training of students and post-doctoral scholars.

\section{First light instrumentation}

The current instrument combines the pre-existing instrumentation of the OVRO and BIMA antennas with the improved sensitivity and imaging provided by having more antennas at a single, higher site. In addition, a major program of cryogenic and control system upgrades has been carried out on the antennas to improve reliability and to reduce routine maintenance.

The CARMA site is Cedar Flat in the Inyo Mountains of California, at 2200 m elevation. The Inyo Mountains are in the rain shadow of the Sierra Nevada range and receive very low precipitation and generally good weather. Operational access is excellent. The fifteen CARMA antennas provide imaging performance superior to other millimeter-wave interferometers through their number and the consequent instantaneous $u v$ coverage and spatial dynamic range. The antennas may be placed in one of five standard configurations (figure 1). The resolution obtainable with the largest "A" configuration at $1 \mathrm{~mm}$ is 0.15 arcsec (table 1), while structures of order an arcminute may be wellimaged with the "E" configuration (8-m baselines) at $3 \mathrm{~mm}$.

A new fiber-optic IF transport system and new spectral line downconverters feed a $1.5 \mathrm{GHz}$ correlator that contains hardware reused from the OVRO (COBRA) correlator. New monitor and control software integrates observing with the project database and archive, and allows dynamic scheduling dependent on the weather conditions. Observing is conducted in "service" mode by CARMA students and postdocs: PIs do not typically need to attend the site. CARMA specifications are given in table 2 .

\begin{tabular}{cccccc}
\hline$\delta$ & $\mathrm{A}$ & $\mathrm{B}$ & $\mathrm{C}$ & $\mathrm{D}$ & $\mathrm{E}$ \\
\hline 30 & $0.15 \times 0.12$ & $0.37 \times 0.30$ & $0.90 \times 0.75$ & $2.2 \times 1.9$ & $4.5 \times 3.9$ \\
0 & $0.15 \times 0.15$ & $0.38 \times 0.37$ & $0.94 \times 0.90$ & $2.4 \times 2.2$ & $5.0 \times 4.4$ \\
-30 & $0.32 \times 0.15$ & $0.75 \times 0.38$ & $1.88 \times 0.92$ & $4.7 \times 2.3$ & $7.6 \times 3.5$ \\
\hline
\end{tabular}

Table 1: FWHM beam size (arcsec) at $230 \mathrm{GHz}$ for $\pm 2 \mathrm{hr}$ transit centered track 


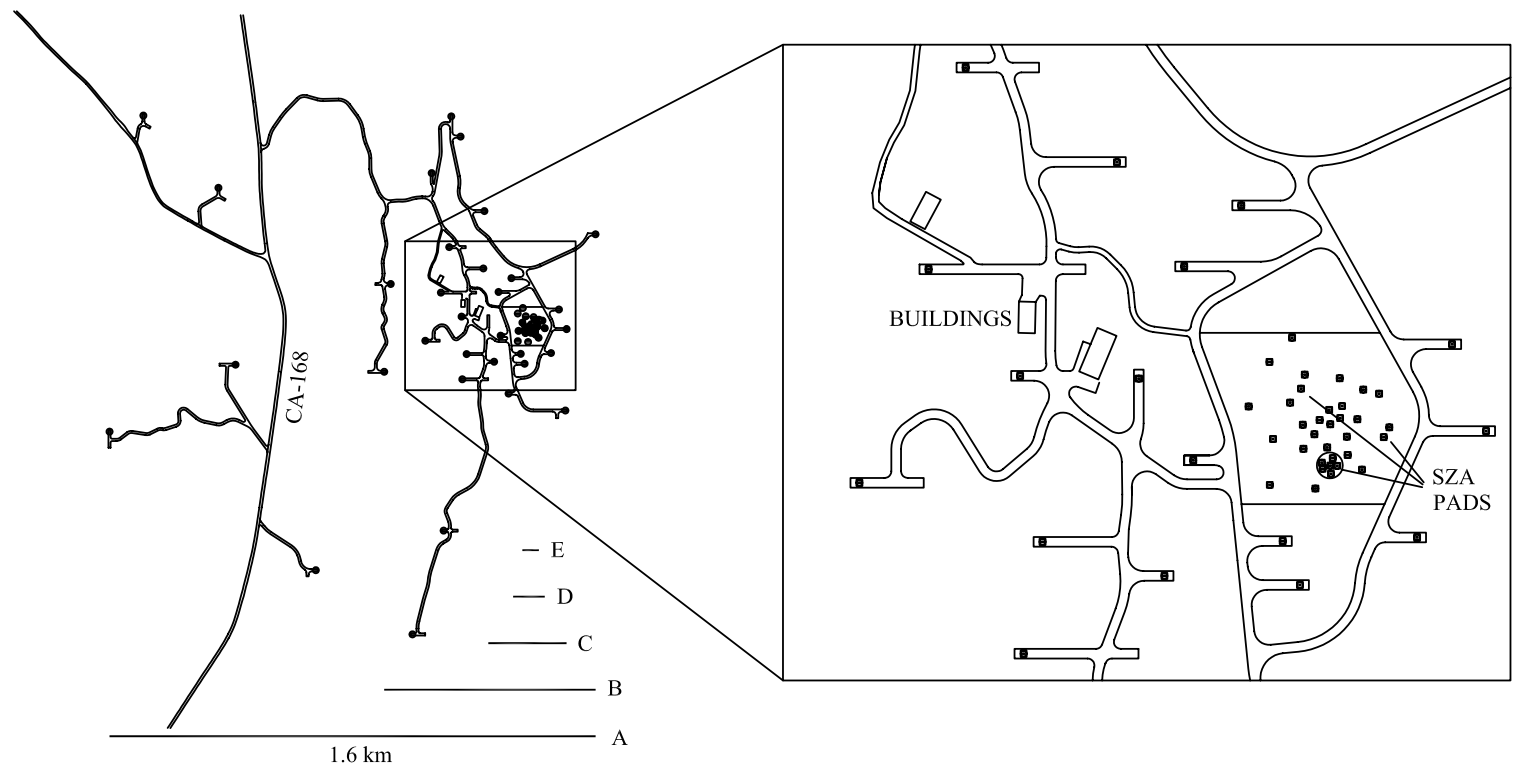

Figure 1: Map of the CARMA site, showing the geographical extent of the five configurations

\section{CARMA science}

A broad range of scientific investigations will benefit from the performance gains stemming from the merger of the arrays, the relocation of the antennas to a higher site, and our continuing program of improvements. Here I present two examples of CARMA's unique millimeter-wave imaging performance.

Wide-field imaging of M51 (The Whirlpool Galaxy) Koda et al. have imaged the entire M51 disk (figure 2) in the $\mathrm{CO}(1-0)$ line with CARMA, and added short spacings with the Nobeyama 45-m antenna. This image has 151 pointings made with the $\mathrm{C}$ and $\mathrm{D}$ configurations. With un-

\begin{tabular}{|c|c|c|}
\hline Parameter & & Value \\
\hline Number of antennas & & $15(6 \times 10.4 \mathrm{~m}, 9 \times 6.1 \mathrm{~m})$ \\
\hline Total collecting area & & $772 \mathrm{~m}^{2}$ \\
\hline Angular resolution & & $0.1^{\prime \prime}$ (230 GHz A configuration) \\
\hline Receiver bands & & $1.3 \mathrm{~mm}, 3 \mathrm{~mm}$ \\
\hline Continuum sensitivity & $\begin{array}{l}100 \mathrm{GHz} \\
230 \mathrm{GHz}\end{array}$ & $\begin{array}{l}0.67 \mathrm{mJy} \mathrm{min}^{-0.5} \\
3.0 \mathrm{mJy} \mathrm{min}^{-0.5}\end{array}$ \\
\hline Line sensitivity $\left(1^{\prime \prime}\right.$ beam, $\left.1 \mathrm{~km} \mathrm{~s}^{-2}\right)$ & $\begin{array}{l}100 \mathrm{GHz} \\
230 \mathrm{GHz}\end{array}$ & $\begin{array}{l}8.2 \mathrm{~K} \mathrm{~min}^{-0.5} \\
4.5 \mathrm{~K} \mathrm{~min}^{-0.5}\end{array}$ \\
\hline Correlator (each sideband) & & $\begin{array}{l}4 \mathrm{GHz} \text { bandwidth, } 5 \mathrm{kHz} \text { resolution } \\
1375 \text { channels }\end{array}$ \\
\hline
\end{tabular}

Table 2: CARMA Specifications. Sensitivities are estimated for the 25th water vapor percentile, once the receiver and correlator upgrades currently underway are complete. 


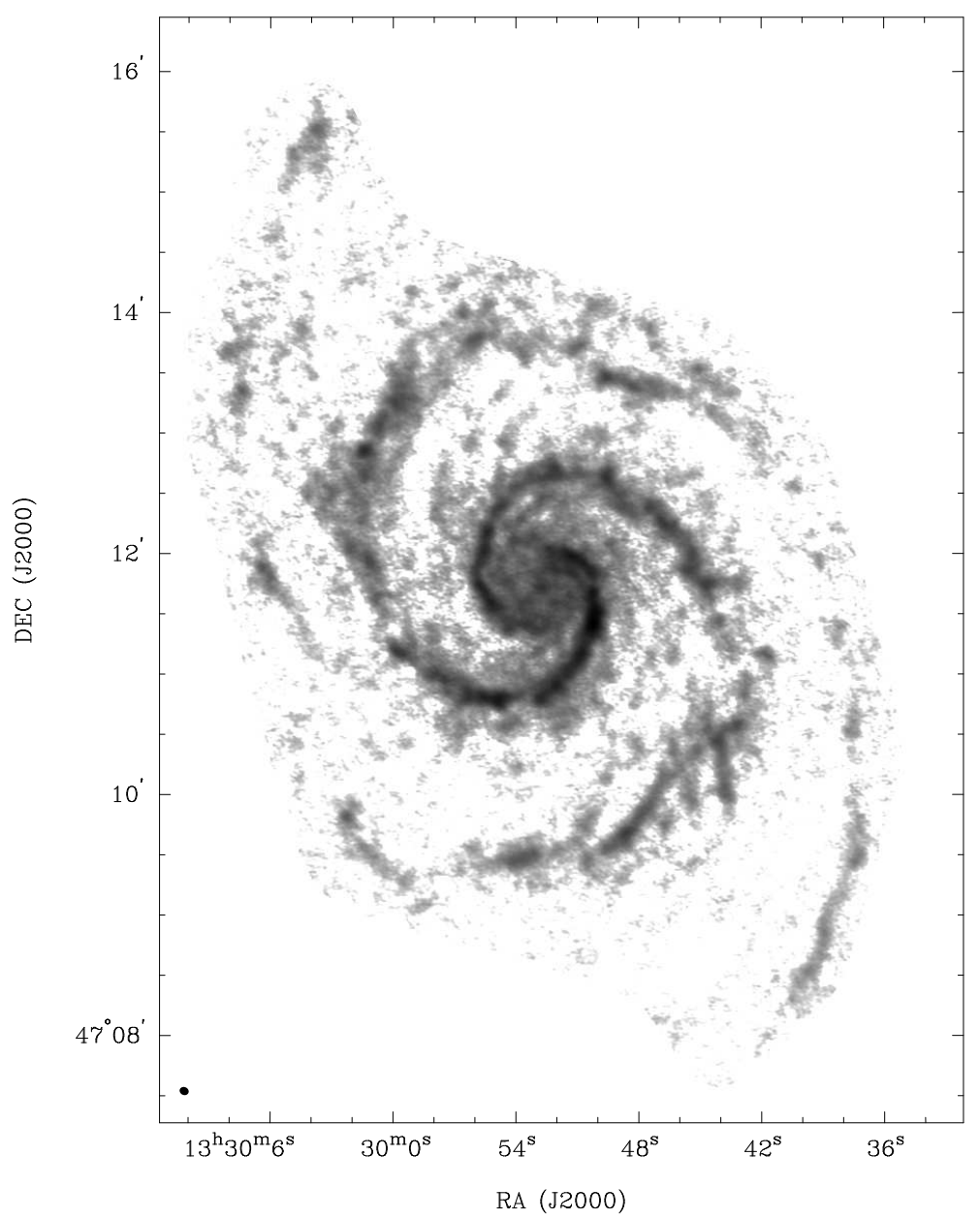

Figure 2: CARMA and Nobeyama integrated CO(1-0) image of M51 (courtesy Jin Koda et al., Caltech). The image is on a logarithmic scale to highlight the low-level structure, including the interarm material. The synthesized beam is shown at the lower left.

precedented image fidelity and spatial resolution, they have detected many giant molecular clouds (GMCs) in both the spiral arms and the interarm regions. Associations of giant molecular clouds (GMAs) are found only in the spiral arms, and thus seem to be unbound, short-lived structures. However, the molecular gas fraction is high even in the interarm regions. Therefore, the GMA destruction is not likely to be caused by stellar feedback, which would destroy molecules as well as GMAs and GMCs.

High-resolution imaging of GG Tau Isella et al. have begun to image a comprehensive set of protoplanetary disks in star-forming regions. Figure 3 shows an image of GG Tau made with the $\mathrm{B}$ and $\mathrm{C}$ configurations at $1 \mathrm{~mm}$ (resolution $0.6 \mathrm{arcsec}$ ). Azimuthal variation in the accretion ring points to locations where planets may form. There are many outstanding questions on the interaction of forming planets with circumstellar material, and on the time scales of formation. It it not clear how many disk produce planetary systems. CARMA will be used to address these problems. 


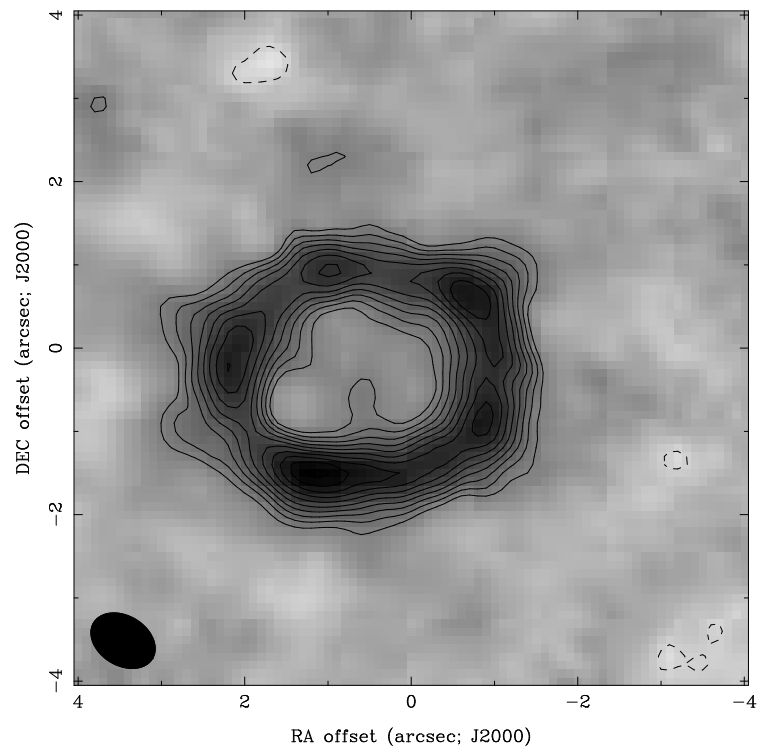

Figure 3: GGTau, combining B and C array configuration data (courtesy of Andrea Isella et al., Caltech). The synthesized beam is shown at the lower left.

\section{Future Developments}

CARMA is continuing a program of performance enhancements. In 2008, we expect to commission new double-sideband mixers at 1 and $3 \mathrm{~mm}$ with $\sim 8 \mathrm{GHz}$ bandwidth. At $1 \mathrm{~mm}$, the new system will be dual polarization. The present correlator will be expanded to eight $500 \mathrm{MHz}$ bands, each reconfigurable with up to 400 channels and channel widths as small as $5 \mathrm{kHz}$. Future enhancements planned include array receivers, a correlator capable of simultaneously handling 2 polarizations and 23 antennas with $\sim 8 \mathrm{GHz}$ bandwidth, a drive upgrade for the 10-m antennas, and an automated imaging pipeline.

\section{Acknowledgments}

CARMA would not have been possible without the efforts of a great many present and former CARMA staff. Support for CARMA construction was derived from the states of California, Illinois, and Maryland, the Gordon and Betty Moore Foundation, the Kenneth T. and Eileen L. Norris Foundation, the Associates of the California Institute of Technology, and the National Science Foundation. Ongoing CARMA development and operations are supported by the National Science Foundation under a cooperative agreement, and by the CARMA partner universities.

\section{References}

[1] W. J. Welch et al., The Berkeley-Illinois-Maryland Association Array, PASP, 108, 93, 1996

[2] S. Padin, S. L. Scott, D. P. Woody, N. Z. Scoville, T. V. Seling, R. P. Finch, C. J. Giovanine, and R. P. Lawrence, The Owens Valley Millimeter Array, PASP, 103, 461, 1991 